\title{
利用超声二次谐波测试水泥试样的非线性系数
}

\author{
谢 凡 ${ }^{1}$ 葛洪魊 $^{2}$ 郭志伟 $^{1}$
}

(1. 中国地震局地震观测与地球物理成像重点实验室 北京 100081 ;

2. 中国石油大学(北京)非常规天然气研究院 北京 102249)

\begin{abstract}
摘要: 使用 RITEC 公司的 RAM5000 非线性测试仪器构建稳定、高可重复性的非线性系数相对观测及数据处理系统。为水 泥制品损伤演化的非线性特征研究提供可靠的观测平台。测量三种不同颗粒直径的水泥试样的名义非线性系数。使用三组高 频换能器对试验样品在 40 组电压激励下产生的高次谐波幅值进行观测。获取减少三次谐波的影响并突出二次谐波的合理的 电压激励参数和数据处理方法。采用线性相关系数作为名义非线性系数稳定性的判断依据。通过 $86.5 \mathrm{~h}, 173$ 次观测验证了 系统的稳定性和可重复性。并获取水泥试样水饱和过程中非线性系数变化和波速变化。
\end{abstract}

关键词: 非线性声学 非线性系数 超声 水泥

中图分类号: TG156

\section{Measurement of Acoustic Nonlinearity Parameter Using Second Harmonics in Cement Specimens}

\author{
XIE Fan ${ }^{1} \quad$ GE Hongkui ${ }^{2}$ GUO Zhiwei ${ }^{1}$ \\ (1. Key Laboratory of Seismic Observation and Geophysical Imaging,Institute of Geophysics, \\ China Earthquake Administration, Beijing 100081; \\ 2. Unconventional Natural Gas Institute, China University of Petroleum, Beijing 102249)
}

\begin{abstract}
A stable, high repeatability acoustic nonlinearity parameter experimental procedures and data process system is deployed to track the damage evolution in cement-based specimens using RAM 5000 of RITEC Inc. Three kinds of cement specimens with different diameters of grain are measured by this system to obtain relative nonlinearity parameters. In order to reduce the influence of third harmonics, highlight the second harmonics, 40 levels of voltages input have been tested by 3 groups of high frequency transducers to observe change of the second and third harmonics combined with pulse-inversion technique usage. Linear correlation coefficients are proposed to evaluate the stability of the system. System stability and repeatability is verified by $86.5 \mathrm{~h}$ within 175 times testing using above mentioned system. Consequently relative nonlinearity parameter change and velocity change of cement specimens are acquired during the process of water saturation.
\end{abstract}

Key words: Nonlinearity acoustics Nonlinearity parameter Ultrasonic Cement-based material

\section{0 前言}

水泥及其制品在基础设施建设中被广泛使用。 对在役期水泥制品的现状的评估也日益受到重视。 由于水泥及其制品内部结构的复杂性以及由此产生 的不同的力学性能 ${ }^{[1]}$, 因此需要比超声波速测量等传 统方法更为敏感无损检测技术对其损伤及其演化定 量化表征。

近来的研究表明 ${ }^{[2]}$, 弹性介质中微裂隙的存在 会打破入射的超声波和接收到的弹性波响应之间的

* 中央级公益性科研院所基本科研业务费专项资助项目(DQJB11B11, DQJB11C04)。20121114 收到初稿, 20130325 收到修改稿
比例关系。其中频率成分的再分布是微结构演化的 主要现象之一。当单周期正弦波在穿越弹性介质时 与介质内部的非线性散射体(诸如微裂隙)相互作用 会导致新的谐波分量的产生。对于水泥及其制品材 料而言, 尽管在宏观上表现出弹性特征, 但其微结 构高度不均质且十分复杂, 呈现非均质特征, 被称 之为非线性细观弹性材料 ${ }^{[3-4]}$ 。表征水泥材料早期退 化特性的微裂隙、微缺陷等的微结构变化与非线性 声学效应密切相关。高阶谐波正是这些非线性声学 现象之一。因此测量水泥及其制品非线性系数的变 化对于揭示水泥介质微结构损伤演化更为敏感 ${ }^{[5]}$ 。

高阶谐波的观测最早开始于金属材料性能的 退化检测。其中 FROUIN 等 $^{[6]}$ 成功的使用了非线性 
超声法跟踪到了铝合金试样的在整个疲劳周期的疲 劳损伤检测, $\mathrm{KIM}$ 等 ${ }^{[7]}$ 介绍了使用非线性系数的绝 对测量方法以及在金属疲劳检测中的应用。SHAH 等 ${ }^{[8-10]}$ 的工作都表明基波和二阶谐波的测量来获取 材料的非线性系数是判断混凝土介质早期损伤状态 有效方法。此外 JHANG 等 ${ }^{[1-12]}$ 对非线性无损检测 的在国际和国内的应用给出了详细的综述。

观测介质非线性系数需要大功率超声谐波发 射系统。RITEC 公司的 RAM-5000 大功率超声谐波 发射系统具有最高激励功率 $5 \mathrm{~kW}$, 峰峰值 $1400 \mathrm{~V}$ 的高功率的射频声脉冲群输出, 并可驱动低效率换 能器。同时具备 $0.1 \sim 200 \mu \mathrm{s}$ 宽的谐波脉冲激励能 力以及可精确测定 RF 脉冲信号的振幅与相位的相 敏超外差接收器, 是理想的观测硬件。

本文使用美国 RITEC 公司的 RAM5000 非线性 测试仪器构建稳定、高可重复性的非线性超声相对 观测及数据处理系统。对不同颗粒直径的水泥试样 的基频和二阶谐波观测, 进而获得非线性系数 $\beta$ 的 相对变化。与之前大部分研究将激发信号的频段集 中在低频域( $\sim 50 \mathrm{kHz})$ 不同, 本文使用对非均质性 细节更加敏感的三对高频探头 $(0.25 \sim 0.50 \mathrm{MHz}$ 、

$0.5 \sim 1.0 \mathrm{MHz} 、 1.0 \sim 2.0 \mathrm{MHz}$ ), 通过 40 组从低到高 电压输入, 获得基频、二次谐波、三次谐波幅值变 化特性并给出了最能突出二次谐波、减少三次谐波 影响的激发电压区间用于非线性系数的拟合。并且 在获取谐波幅值的过程中采用了脉冲反转技术来辨 识二次谐波。

测量的稳定性和可重复性在任何观测研究工 作中都值得重视, 尤其对介质细微结构变化更为敏 感的非线性系数测量。因此, 本文针对 RAM5000 只能进行单电压手动激发的不足进行改进, 采用 LabVIEW 对 RAM5000 激发端参数进行控制, 构建 了无需人工干预的自动化观测流程。采用线性相关 系数作为非线性系数稳定性的判断依据。通过 86.5 h, 173 次观测验证了系统的稳定性和可重复性。最 后, 本文对水泥试样在水饱和过程中的非线性系数 的变化及波速变化进行了监测。通过本文的工作可 为今后研究水泥及其制品损伤的早期演化的非线性 特征研究提供可靠的观测系统和方法。

\section{1 谐波产生原理}

根据非线性应力-应变本构关系可得到二阶的
一维非线弹性波动方程为

$$
\frac{\partial^{2} u}{\partial t} \approx c^{2} \frac{\partial^{2} u}{\partial x^{2}}\left(1+\beta \frac{\partial u}{\partial x}\right)
$$

式中, $c$ 为介质波速; $t$ 为时间; $u$ 为质点振动位移; $\beta$ 为二阶弹性系数, 即非线性系数; $x$ 是波传播的 距离。运用微扰理论得到非线性运动方程的解为

$$
u=u_{0}+u^{\prime}=A_{1} \cos (k x-w t)+A_{2} \cos [2(k x-w t)]
$$

式中, $u_{0}$ 为线性方程解; $u^{\prime}$ 代表非线性解; $A_{1}$ 为接 收端基频(激发频率)的振幅, $A_{2}$ 为产生的二次谐波 振幅, 其大小由非线性系数 $\beta$ 决定, 满足

$$
A_{2}=\frac{\beta}{8} A_{1}^{2} k^{2} x
$$

式中, $k$ 为超声波波数, $k=2 \pi / \lambda ; \lambda$ 为超声波波 长。相应地, 非线性系数 $\beta$ 可通过测量接收端基频 和二次谐波的振幅并计算得到

$$
\beta=\frac{8}{k^{2} x} \frac{A_{2}}{A_{1}^{2}}
$$

由式(4)可知, 在测量非线性系数时, 非线性系 数不仅与基频振幅以及二次谐波振幅有关, 还与超 声波波数和传播距离有关。通常测量中超声波激发 频率和传播距离不变, 则 $A_{2} / A_{1}^{2}$ 非线性系数成正比。 同理可得到三阶非线性系数 $\beta_{2}$ 与三次谐波振幅的 关系

$$
\beta_{2}=\frac{32}{k^{4} x^{2}} \frac{A_{3}}{A_{1}^{3}}
$$

式(4)中, 存在将电压信号转换成位移信号、考虑超 声在水泥材质转播的漫射、散射等情况的衰减系数、 以及测试系统耦合标定等所诸多难点问题, 在实际 工作中可通对非线性系数的相对观测, 即测量不同 频率段电压信号的功率谱幅值获得名义非线性系 数, 即

$$
\beta^{\prime}=\frac{V(2 \omega)}{V^{2}(\omega)}
$$

\section{2 试验研究}

\section{1 试验试样的制备}

本文制作了骨料颗粒直径不同的水泥试样若 干块。水泥试样的具体参数如表 1 所示。制作过程 中为了尽量消除水泥中生成的气泡, 采用了水泥消 
泡剂(实际试样中仍然存在少量气泡)。试样制备完 成以后均经过 $28 \mathrm{~d}$ 的养护。波速测量采用脉冲法并 取 10 次测量的平均值。

表 1 三种水泥试样的物理参数

\begin{tabular}{cccccc}
\hline & & & & \\
编号 & 长×宽×高 & $\begin{array}{c}\text { 骨料颗粒 } \\
\text { (质量) }\end{array}$ & 粒径 $/ \mathrm{cm}$ & $\begin{array}{c}\text { 传播 } \\
\text { 时间 } / \mu \mathrm{s}\end{array}$ & 波速 $/(\mathrm{m} / \mathrm{s})$ \\
\hline 1 & $10 \times 10 \times 10$ & $0.4: 1: 1$ & $0.1 \sim 0.2$ & 24.2 & 4386 \\
2 & $10 \times 10 \times 10$ & $0.4: 1: 1$ & $0.3 \sim 0.6$ & 23.1 & 4424 \\
3 & $10 \times 10 \times 10$ & $0.4: 1: 1$ & $0.8 \sim 1.2$ & 22.0 & 4506 \\
\hline
\end{tabular}

2.2 试验仪器及系统

非线性超声测试系统的结构图如图 1 所示。

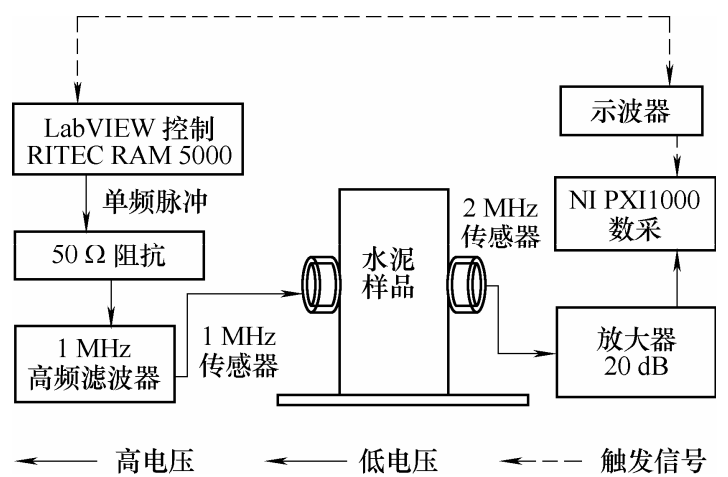

图 1 试验流程示意图

发射端使用 RAM5000 系统。单频正弦波经过 $50 \Omega$ 的阻抗和滤波器组后输入水泥试样一端的发 射换能器。水泥试样置于泡沫塑料板上以隔绝桌面 传导的影响。发射换能器和接收换能器均为 $\mathrm{P}$ 波换 能器, 一共 4 个分为 3 对使用。分别是第一组 0.25 $\mathrm{MHz}$ 激发 $0.5 \mathrm{MHz}$ 接收; 第二组 $0.5 \mathrm{MHz}$ 激发 1 $\mathrm{MHz}$ 接收; 第三组 $1 \mathrm{MHz}$ 激发 $2 \mathrm{MHz}$ 接收。表 2 给出了 4 种超声换能探头的具体参数。其中 $3 、 4$ 号铌酸锂换能器由 RITEC 公司提供并推荐使用。接 收端使用接收换能器接收低电压超声信号并由 NI 数采系统以 $20 \mathrm{MHz}$ 的采样率采集并进行 60 次叠加 后进行后期处理。整个观测系统触发信号采用 RM5000 的外部触发信号与示波器外触信号相连并 与 NI PXI1000 触发端相连。通过 LabVIEW 数据采 集程序控制发射端和接收端按给定模式自由调整激 发参数和接收参数。

表 24 种超声换能探头

\begin{tabular}{cccc}
\hline 编号 & 主频 & 材质/型号 & 类型 \\
\hline 1 & $0.25 \mathrm{MHz}$ & PAC Nano & 宽带 \\
2 & $0.50 \mathrm{MHz}$ & 泛美 A101S & 窄带 \\
3 & $1 \mathrm{MHz}$ & Lithium Niobate & 窄带 \\
4 & $2 \mathrm{MHz}$ & Lithium Niobate & 窄带 \\
\hline
\end{tabular}

\section{3 试验方法}

为保证超声波延单一方向传播并尽量减少衰 减及边界效应，发射的正弦波的周期长度与水泥试 样的长度相当。激发电压采用 RM5000 的电压模块 控制，一共发射 40 组，电压范围 $10 \sim 915 \mathrm{~V}$ ，分别 由 RAM5000 中的输出振幅百分比 Amplitute(\%-FS) 和门控放大器 Output Level 控制。其中前 10 组为低 压: Output Level 为零后, Amplitude 从 $10 \%$ 到 $100 \%$ ，步长 $10 \%$ 。电压范围 $10 \sim 100 \mathrm{~V}$ 。后 30 组 为高压: 在 Amplitude 为 100\%后, Output Level 从 2 到 60 , 步长为 2 , 电压范围 $114 \sim 915 \mathrm{~V}$ 。每组电 压分别在 0 相位和 180 相位发射以便于使用脉冲反 转技术对二次谐波进行识别。

三对换能器与试样耦合均采用轻质润滑油 ${ }^{[7]}$ 并使用水杨酸本脂行固定。换能器分别安装在试 样两端的中心位置。数据采集系统使用美国国家 仪器公司的 PXI1000 系列。采用 LabVIEW 编制 采样程序, 设定采样率为 $20 \mathrm{M} / \mathrm{s}$, 采样长度为 5000 个点。

在后期数据处理中使用脉冲反转技术增强对 二次谐波识别。脉冲反转技术可有效增强偶次波。 关于脉冲反转技术可以参考文献[14]。如图 2 所 示, 在电压 Amplitude 为 $100 \%$, Output Level 为 0 下, 采用 $1 \mathrm{MHz}$ 激发, $2 \mathrm{MHz}$ 接收的铌酸锂 换能器在 $0^{\circ}$ 相位和 $180^{\circ}$ 相位接受到的时域信 号。为减少散射、衰减等情况对高次谐波的干扰, 取与直达波长度相当的 500 个点 $(25 \mu \mathrm{s})$ 进行傅里 叶变换，如图 3 所示，虚线所表示的原始波形的 频谱图上基频很清楚地得到，但是二次谐波并不 明显。通过脉冲反转技术，实线所表示的二次谐 波的幅值得到凸显。通过这种方法可以有效地提 高二次谐波分辨率。

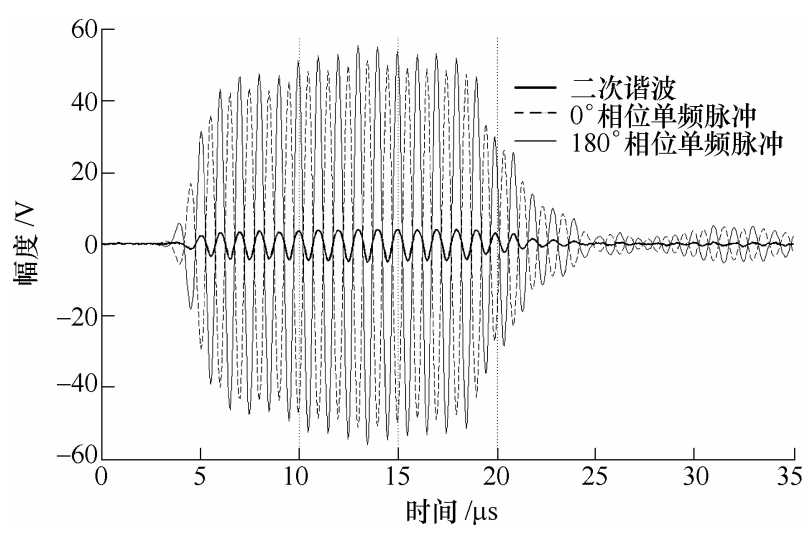

图 2 穿过水泥试样的单频脉冲时域信号 


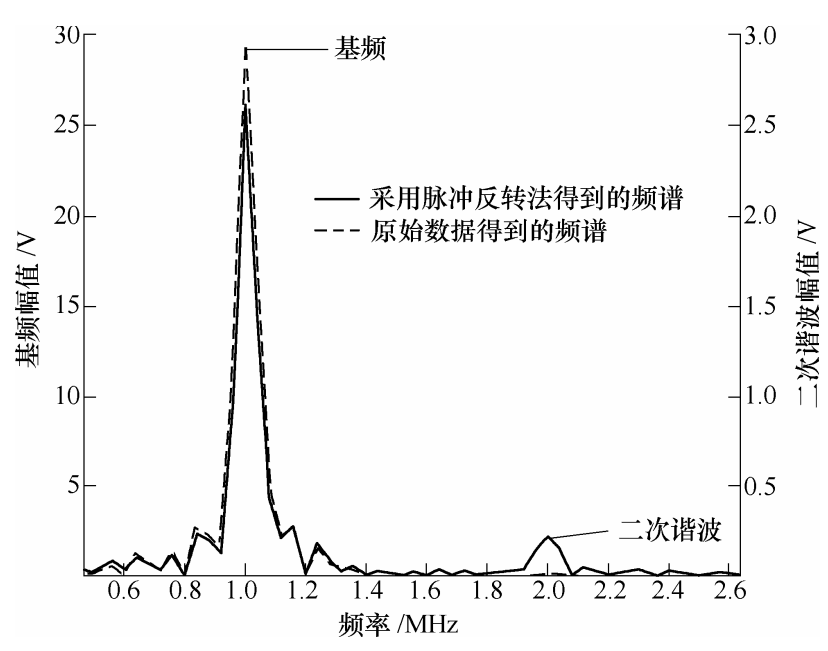

图 3 原始时域信号和使用脉冲反转技术得到的

\section{4 试验结果}

二次谐波频谱图

在研究非线性声学现象过程中, 由于采用的大功 率超声技术, 因此需要选取适当的激发电压。当电压 激发过低时, 无法得到高分辨率的二次谐波; 当电压 输入过高时会出现更高次谐波, 更高次谐波的出现使 得水泥的非线性声学特征更为复杂。为避免这一现象 并获得以二次谐波为主的非线性系数, 需要从 $10 \mathrm{~V}$ 到 $915 \mathrm{~V}$ 一共 40 组激发电压中优选出既能突出二次谐波 又能减少三次谐波影响的激发电压区间。

图 4 6 分别是 40 组激发电压下, 三组不同频 段的换能器观测到的三种不同颗粒直径的水泥试样 的基频幅值和二阶谐波及三阶谐波的幅值。图 4a、 $5 \mathrm{a} 、 6 \mathrm{a}$ 可以看出, 基频的平方与二次谐波幅值的比

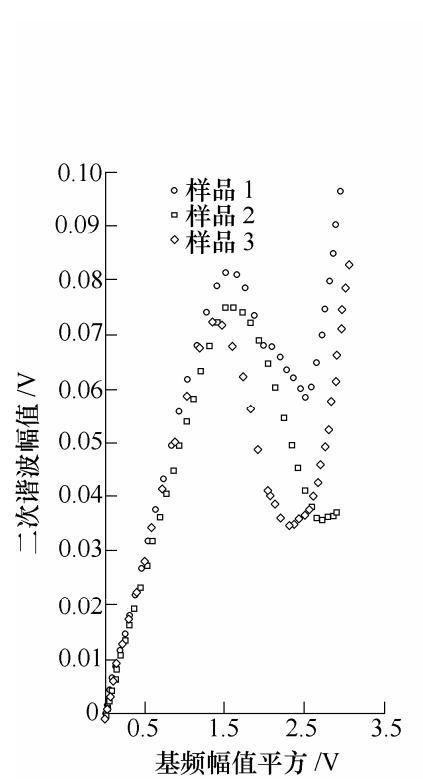

(a) 基频幅值的平方与二次谐波 幅值之比

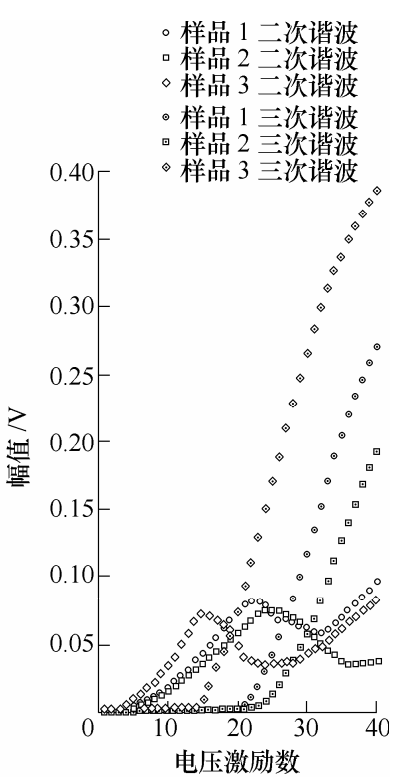

(b) 二次谐波幅值与三次 谐波幅值
图 4 第一组换能器 $(0.25 \sim 0.50 \mathrm{MHz})$ 随激励电压增大谐波 幅值的变化
值在前 15 次电压激发(大约从 $10 \sim 170 \mathrm{~V}$ )中都保持 了比较好的线性上升的趋势, 随后该比值出现下降 并伴随波动, 然后重新上升, 在高电压情况 $(>170 \mathrm{~V})$ 下线性度并不理想。图 $4 \mathrm{~b} 、 5 \mathrm{~b} 、 6 \mathrm{~b}$ 可以看出, 在前 15 次测量中, 二次谐波的幅值均大于三次谐波且三 次谐波增长缓慢, 随后三次谐波的幅值迅速增大并 在最后 10 次激发中数倍于二次谐波的增幅。可见， 在观测二次谐波中，合理的电压激励幅值必须予以 考虑。在本次水泥试样的二次谐波的观测中, 为避 免电压过低导致分辨率不足，更为避免电压过高导 致三次及高次谐波对非线性系数观测的干扰，选择 输出振幅百分比 Amplitute(\%-FS)从 50\%到 100\%, 门控放大器 Output Level 从 2 到 10, 电压幅值从 50 $\mathrm{V}$ 到 $175 \mathrm{~V}$ 的 10 个电压输出作为二次谐波观测输入 电压。

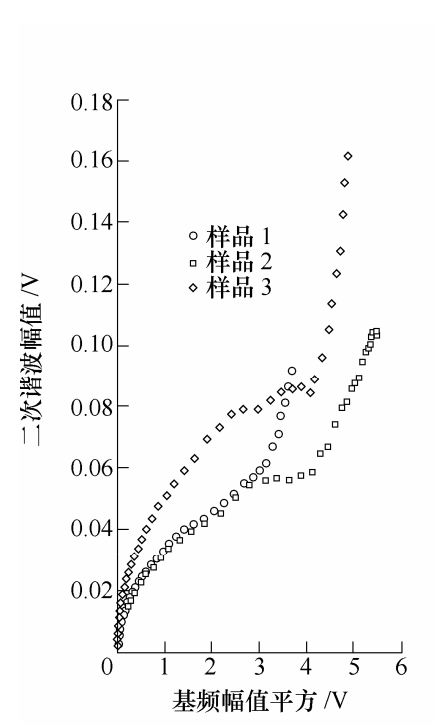

(a) 基频幅值的平方与二次谐波 幅值之比

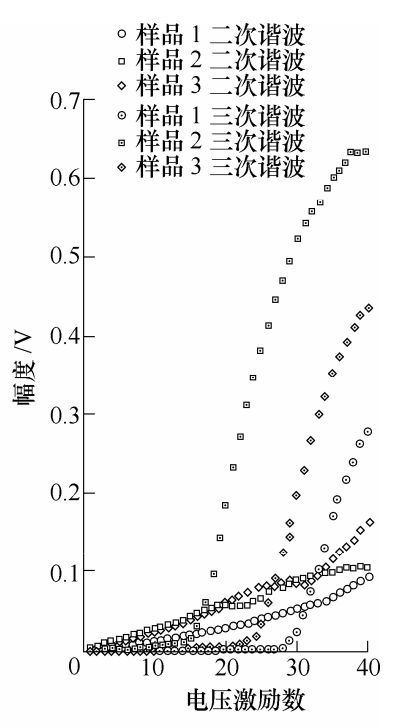

(b) 二次谐波幅值与三次 谐波幅值
图 5 第二组换能器 $(0.5 \sim 1.0 \mathrm{MHz})$ 随激励电压增大谐波 幅值的变化

通过图 4a、5a、6a 中线性度较好的前 15 次激 发样品斜率分析可知, 大颗粒直径的 3 号水泥试样 的非线性系数(即斜率)较大。特别是图 5a、6a 中, 其与小颗粒直径的 1 号、2 号样品和区分度非常明 显; 而颗粒直径比较接近的 1 号、2 号样品的非线 性系数(即斜率)则较为接近; 从三组不同频率的换 能器角度看, 图 $6 \mathrm{a}$ 非线性系数区分度最为清晰, 而 图 4a、5a 中小颗粒直径水泥试样的非线性系数可分 辨程度依次下降。

测量对介质细微变化敏感的非线性系数对系 统稳定性以及测量结果的可重复性要求更为严格, 


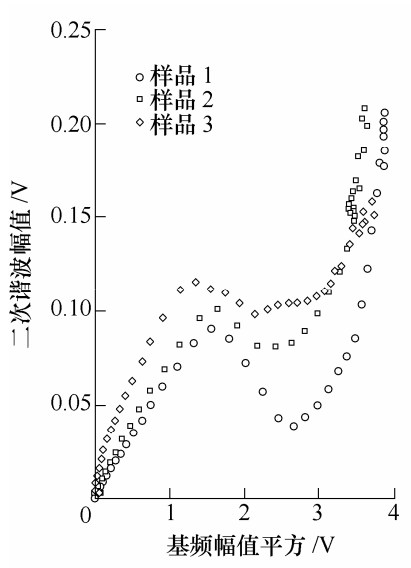

(a) 基频幅值的平方与二次谐波

幅值之比

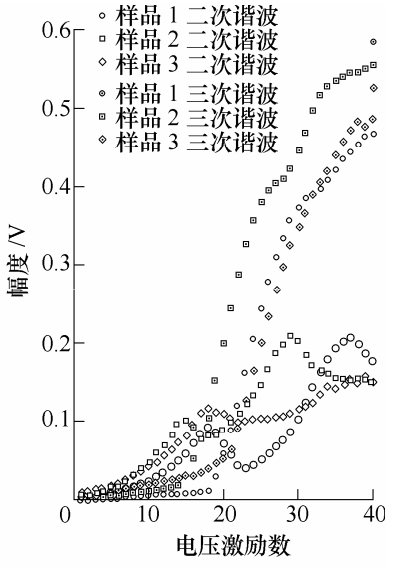

(b) 二次谐波幅值与三次

谐波幅值
图 6 第三组换能器 $(1 \sim 2 \mathrm{MHz})$ 随激励电压增大谐波 幅值的变化

因此本文对二次谐波观测的稳定性和可重复性进行 了试验验证。具体来说, 使用 $1 \mathrm{MHz}$ 发射 $2 \mathrm{MHz}$ 接收的铌酸锂换能器每隔 $30 \mathrm{~min}$ 对 3 号水泥试样激 发 10 个电压(Amplitute( $\%$-FS)从 $50 \%$ 到 100\%, 门控 放大器 Output Level 从 2 到 10 , 电压幅值从 $50 \mathrm{~V}$ 到 $175 \mathrm{~V}$ ) 作为二次谐波观测的激发电压, 一共观测 173 次。通过最小二乘拟合获得二次谐波与基频幅 值平方的线性关系。最后使用互相关法得到观测值 与拟合直线的线性相关系数并以此作为系统是否稳 定的指标。

图 7 为第 173 次观测的结果。在该次观测中, 观测值与拟合后的二次谐波与基频幅值平方的相关 系数达到 0.9976 。图 8 给出了 173 次测量的线性度 相关系数的均值是 0.9988 , 方差仅为 0.0016 。因此 通过长时间的重复观测相关系数的均值可知该观测 系统有较高的稳定性和可重复性。

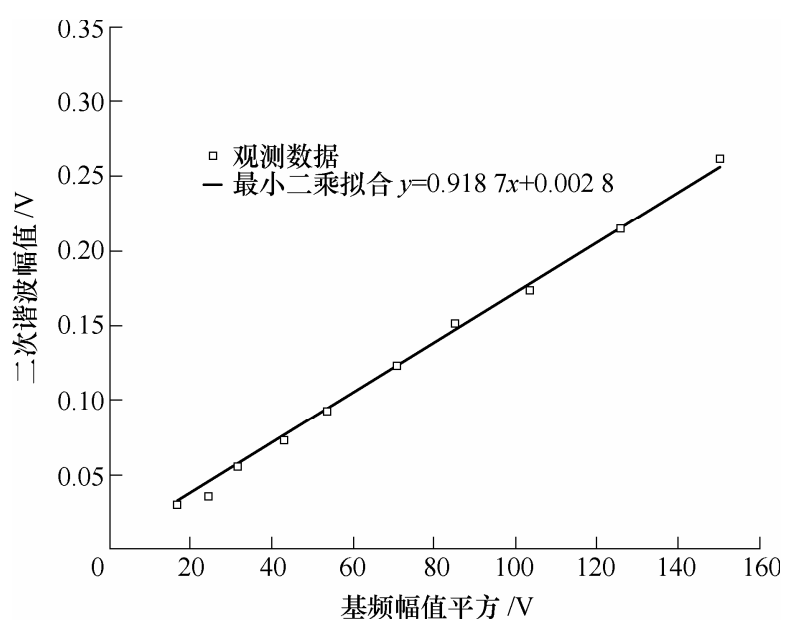

图 7 随电压幅值增大的基频幅值的平方与 二次谐波幅值之比

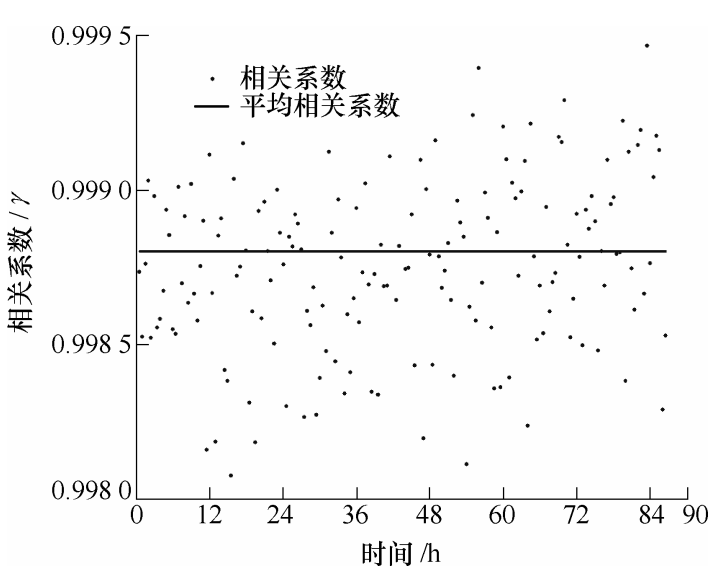

图 8173 次测量的线性度相关系数

\section{3 水饱和过程中的非线性系数变化}

本文对颗粒直径为 $0.8 \sim 1.2 \mathrm{~cm}$ 的 3 号水泥试样 进行了水饱和过程监测。水泥试样放置于装入纯净 水的塑料水桶中，水桶底部铺设塑料垫片，水线不 超过换能器布设的位置, 如图 9 所示。通过水泥试 样本身的吸附能力,使得其水线不断上升, 含水量不 断增加。试验使用 $1 \mathrm{MHz}$ 发射 $2 \mathrm{MHz}$ 接收的铌酸 锂换能器每隔 $30 \mathrm{~min}$ 对 3 号水泥试样激发 10 个电 压(Amplitute $(\%-\mathrm{FS})$ 从 $50 \%$ 到 $100 \%$, 门控放大器 Output Level 从 2 到 10, 电压幅值从 $50 \mathrm{~V}$ 到 $175 \mathrm{~V}$ ) 作为二次谐波观测的激发电压。最后激发一个周期 的电压(Amplitute 100\%, 门控放大器 Output Level 0) 进行波速测量。声波到时拾取采用互相关法，即先 手动识别一个波形到时作为参考波形，其余波形由 自动识别算法计算并通过与参考波形的互相关得到 校正，波速测量相对误差小于 $1 \%$ 。

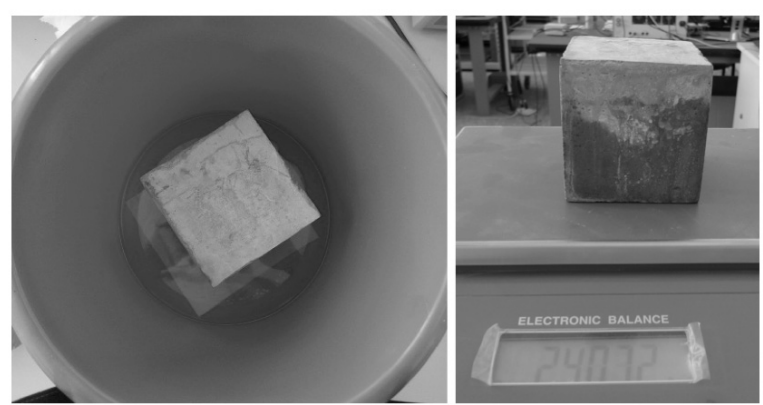

图 9 水泥试样水饱和监测示意图

该试验一共观测 38 次 $(19 \mathrm{~h})$ 。非线性系数线性 相关系数均值是 0.988 , 方差 0.0019 。水泥试样初始 干燥质量为 $2389.5 \mathrm{~g}$, 观测完毕后质量 $2407.2 \mathrm{~g}$, 增重 $17.7 \mathrm{~g}$ 。非线性系数的变化和波速变化如图 10 所示。 


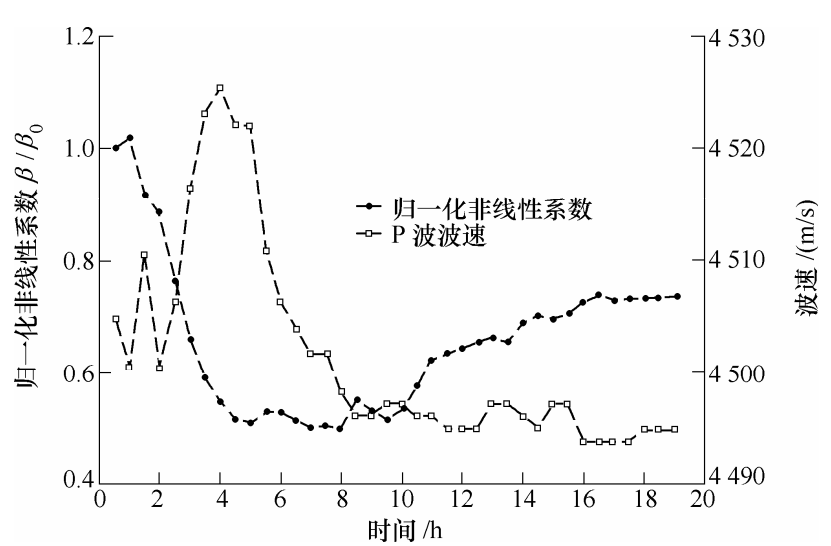

图 10 水饱和过程中的非线性系数和波速的变化

图 10 中, 水泥试样水饱和过程的前 $5 \mathrm{~h}$, 非线 性系数在降低, 而波速在增大, 随后波速快速下降 而非线性系数则趋于稳定, $10 \mathrm{~h}$ 后直到第 $19 \mathrm{~h}$ 波速 缓慢下降而非线性系数则有一定程度增加。

\section{4 讨论}

(1) 本文对非线性系数的测量是动态测量法。 目前的一些研究显示 ${ }^{[15-16]}$, 采用随应力改变的尾波 干涉技术 ${ }^{[17]}$ 亦可求取介质的非线性系数, 即

$$
\beta=-\frac{E}{v^{0}} \frac{\partial v}{\partial \sigma}
$$

式中, $E$ 为弹性模量; $-\frac{1}{v^{0}} \frac{\partial v}{\partial \sigma}$ 则代表了波速变化 与应力 $\sigma$ 的斜率关系。

今后的研究中用有必要对这两种方法求取介 质非线性系数做更进一步的探讨。

(2) 高频换能器的使用对于粒径间的早期损伤 的微裂隙的探测理论上更为敏感, 但水泥制品内部 结构复杂。表现在三次及以上的高阶谐波随着激励 电压的增大而增大的特征非常明显。而高次谐波通 常与材料的高阶弹性常数密切相关。这使研究水泥 等复杂介质的以二阶谐波为代表的经典非线性现象 中需要考虑更高次的非线性声学现象的影响。

\section{5 结论}

(1) 本文以 RITEC 公司的 RAM5000 仪器为主 构建了通过以二阶谐波与基频谐波平方的幅值比观 测水泥试样非线性系数的相对测量系统。并对三种 不同颗粒直径的水泥试样进行了名义非线性系数的 测量。为了减少三次谐波的影响, 突出二次谐波, 使用三组高频换能器对不同激励电压、频率下的基 频、二次谐波和三次谐波幅值进行观测。结果显示, Amplitute( $\%$-FS)从 $50 \%$ 到 100\%, 门控放大器 Output
Level 从 2 10, 电压幅值从 $50 \sim 175 \mathrm{~V}$ 的 10 个电 压能够获得可靠的二次谐波; 高频换能器 $(1 \mathrm{MHz}$ 发射 $2 \mathrm{MHz}$ 接收的铌酸锂换能器)对不同颗粒直径 的水泥试样非线性系数变化敏感; 使用脉冲反转技 术能够获得高辨识度的二次谐波。

(2) 对 3 号水泥试样 $86.5 \mathrm{~h}, 173$ 次非线性系数 观测验证了本系统的稳定性和可重复性。给出了使 用拟合后的直线与测量值的线性相关系数线作为稳 定性的判断依据。通过 173 次测量获得的线性相关 系数的均值是 0.9988 , 方差仅为 0.0016 。

(3) 监测了水饱和过程中 3 号水泥试样的非线 性系数与波速随时间变化。发现前 $5 \mathrm{~h}$, 非线性系数 在降低，而波速在增大，随后波速快速下降而非线 性系数则趋于稳定, $10 \mathrm{~h}$ 后一直到第 $19 \mathrm{~h}$ 波速缓慢 下降而非线性系数则有一定程度增加。

研究水泥材料内部微结构的非线性超声谐波 特征的规律为不仅在判断和预测损伤程度和过程等 方面具有重要的实用价值，更可在损伤触发机制以 及损伤后介质随时间恢复过程等科学问题的理解中 发挥重要作用。

\section{参 考 文 献}

[1] MEHTA P K. Concrete : Structure, properties and materials[M]. NJ: Prentice-Hall, 1996.

[2] ANTONACI P, BRUNO C L E, GLIOZZI A S, et al. Monitoring evolution of compressive damage in concrete with linear and nonlinear ultrasonic methods[J]. Cement and Concrete Research, 2010, 40(7): 1106-1113.

[3] GUYER R A, JOHNSON P A. Nonlinear mesoscopic elasticity: The complex behaviour of granular media including rocks, concrete, soil[M]. New York: Wiley, 2009.

[4] JOHNSON P. The new wave in acoustic testing[J]. Journal of the Institute of Materials, 1999, 7(9): 544-546.

[5] ANTONACI P, BRUNO C L E, BOCCA P G, et al. Nonlinear ultrasonic evaluation of load effects on discontinuities in concrete[J]. Cem. Concr. Res., 2010, 40(2): 340-346.

[6] FROUIN J, SATHISH S, MATIKAS T E, et al. Ultrasonic linear and nonlinear behavior of fatigued Ti-6Al-4V[J]. J. Mater. Res., 1999,14: 1295-1298.

[7] KIM J Y. Experimental characterization of fatigue damage in a nickel-based super alloy using nonlinear ultrasonic wave[J]. J. Aeoust. Soc. Am., 2006, 120(3): 1266-1273.

[8] SHAH A, REBAKOV Y. Non-destructive evaluation of concrete in damaged and undamaged states[J]. Materials 
and Design, 2009, 30(9): 3504-3511.

[9] 朱金林, 刘晓宙, 周到, 等. 声波在有裂纹的固体中的 非经典非线性传播 [J]. 声学学报, 2009, 34(5): 234-241.

ZHU Jinlin, LIU Xiaozhou, ZHOU Dao, et al. Nonclassical nonlinear ultrasound transmission in solid with cracks[J]. ACTA Acoustica, 2009, 34(5): 234-241.

[10] 陈小佳, 沈成武, JACOBS L L. 一种基于非线性超声 谐波幅值比的微裂缝探测方法[J]. 武汉大学学报, 2007, 40(6): 61-65.

CHEN Xiaojia, SHEN Chengwu, JACOBS L L. A method for microcrack detection based on harmonic amplitude ratio of nonlinear ultrasonics[J]. Engineering Journal of Wuhan University, 2007, 40(6): 61-65.

[11] JHANG K Y. Nonlinear ultrasonic techniques for non-destructive assessment of micro damage in material:

A review[J]. International Journal of Precision Engineering and Manufacturing, 2009, 10(1): 123-135.

[12] 周正干, 刘斯明. 非线性无损检测技术的研究, 应用和 发展 $[\mathrm{J}$. 机械工程学报, 2011,47(8): 2-10.

ZHOU Zhenggan, LIU Siming. Nonlinear ultrasonic techniques used in nondestructive testing: A review[J].
Journal of Mechanical Engineering, 2011, 47(8): 2-10.

[13] RETEC Inc. RETEC SNAP RM5000 Users Manual Version[M] . Leicestershire: RETEC Inc, 2008.

[14] MUELLER T. Characterization of microstructure evolution of nickel-base superalloys using nonlinear ultrasonic techniques[D]. Georgia: Georgia Institute of Technology, 2005.

[15] ERIC L, STEPHEN H. Monitoring stress related velocity variation in concrete with $2 \times 10^{-5}$ relative resolution using diffuse ultrasound[J]. The Journal of the Acoustical Society of America, 2009,125: 2641.

[16] SCHURR D P. Monitoring damage in concrete using diffuse ultrasonic coda wave interferometry[D]. Georgia: Georgia Institute of Technology, 2010.

[17] AKI K. Analysis of the seismic coda of local earthquakes as scattered waves[J]. Journal of Geophysical Research, 1969, 74(2): 615-631.

作者简介: 谢凡, 男, 1981 年出生, 博士。主要研究方向为介质变化 的高精度检测技术, 超声及声发射试验。

E-mail: xiefan@cea-igp.ac.cn 